\title{
Pancreatic cancer adjuvant radiotherapy target volume design: based on the postoperative local recurrence spatial location
}

Wei Yu', Wei Hu${ }^{1}$, Yongjie Shui ${ }^{1}$, Xiaoyang Zhu' ${ }^{1}$, Chao Li ${ }^{1}$, Xiaoqiu Ren ${ }^{1}$, Xueli Bai ${ }^{2}$, Risheng Yu ${ }^{3}$, Li Shen ${ }^{1}$, Tingbo Liang ${ }^{2}$, Lei Zheng ${ }^{4}$ and Qichun Wei ${ }^{1,5^{*}}$

\begin{abstract}
Objectives: To explore the areas at highest risk for postoperative pancreatic cancer local recurrence according to the spatial location of local failures, with the aim to provide a precise target volume for pancreatic cancer adjuvant radiotherapy.

Methods: Patients with pancreatic cancer who had undergone surgery for the primary tumor in pancreas at our institution from January 2010 to August 2015 were retrospectively analyzed. All local recurrences were plotted on the computed tomography $(C T)$ image of a representative patient according to their relative coordinates to superior mesenteric artery (SMA) or celiac axis (CA). Adjuvant radiation clinical target volume (CTV)-90 and CTV-80 were created to cover $90 \%$ and $80 \%$ plotted recurrences. This planning approach was applied in four simulated cases with comparison to the plan according to RTOG 0848 contouring consensus guidelines. Raystation v4.5.1.14 was used for analyzing high throughput physics data.
\end{abstract}

Results: Eighty-three patients with local recurrence were included from 305 postoperative pancreatic cancer patients who did not receive adjuvant radiotherapy. Thirty-one (37 \%) patients did not have adjuvant therapy at all, 52 (63\%) patients undergone adjuvant chemotherapy alone. Spatial location of local failure was created. Most recurrences occurred near CA or SMA. CTV-90 was generated through expanding the combined SMA and CA contours by $30 \mathrm{~mm}$ right-lateral, $21 \mathrm{~mm}$ left-lateral, $20 \mathrm{~mm}$ anterior, $13 \mathrm{~mm}$ posterior, $10 \mathrm{~mm}$ superior, and $20 \mathrm{~mm}$ inferior. CTV-80, smaller in volume, was also created for simultaneous integrated boost. Through comparison and analysis of the simulated cases, the radiation volumes proposed were much smaller than those with RTOG 0848 contouring consensus guidelines (average volume: PTV-80 $=120 \mathrm{ml}$, PTV-90 $=220 \mathrm{ml}$, RTOG PTV $=490 \mathrm{ml}$ ). Accordingly, the organs at risk received less irradiation dose with the proposed CTV-90 and CTV-80.

Conclusions: Smaller adjuvant radiotherapy CTVs targeting the high-risk local failure areas of postoperative pancreatic cancer were proposed, according to the three-dimensional spatial location of local recurrences. This may help to minimize radiation-related toxicities, achieve dose escalation, and finally reduce local recurrence.

Keywords: Pancreatic cancer, Local recurrence pattern, Spatial location, Target volume, Adjuvant radiotherapy

\footnotetext{
* Correspondence: Qichun_Wei@zju.edu.cn

'Department of Radiation Oncology, the Second Affiliated Hospital, Zhejiang University School of Medicine, Hangzhou, Zhejiang 310009, People's Republic of China

${ }^{5}$ Department of Radiation Oncology, the Second Affiliated Hospital, Ministry of Education Key Laboratory of Cancer Prevention and Intervention, Zhejiang University School of Medicine, Jiefang Road 88, Hangzhou 310009, People's Republic of China

Full list of author information is available at the end of the article
} 


\section{Introduction}

Pancreatic cancer (PCA) is the fourth deadliest solid malignancies in the United States. It is estimated that 48960 new cases will be diagnosed as PCA in the USA in 2015. Among them, 40560 will die from this disease [1]. In China, PCA is the 9th most commonly diagnosed cancer and the sixth leading cause of cancer death. The National Central Cancer Registry (NCCR) of China predicted that there will be approximately 90100 newly diagnosed pancreatic cancer cases and 79400 cases will die from this disease in 2015 [2]. Resectability of the cancer is important to stratify survival. However, at the time of diagnosis, only $20 \%$ of patients are able to undergo surgical resection. Even in these patients, the 5 years overall survival rate is only $10-20 \%$. Although most of patients died from distant metastases, it has been verified that the incidence of local recurrence were up to $20 \%$ to $60 \%$ [3-5], and autopsy studies reported even higher rates of local recurrence [6]. These findings have highlighted the importance of local control in resectable pancreatic cancer.

The standard options of adjuvant therapy for pancreatic cancer include chemotherapy and chemoradiation (CRT). However, the role of radiation therapy in the adjuvant therapy is still controversial [7-12]. One of the explanations is the insufficient of radiation dose in conventional radiotherapy, due to the normal structure dose constraints. Furthermore, the integration of approximately 6 weeks of chemoradiation (CRT) (45 Gy directed to the tumor bed, surgical anastomoses and peripancreatic nodes with boost of 5 to 15 Gy to the tumor bed) has high toxicity which decreases quality of life and delays the delivery of full dose chemotherapy [7].

Nevertheless, technological developments in image guidance and motion management have enabled the precise radiotherapy. So smaller target volume is possible in order to minimize treatment-related toxicity. Stereotactic body radiotherapy (SBRT) represents a novel field of radiation therapy and is a tumor-ablative radiation modality employing multiple non-coplanar fixed beams or arc fields to damage the target accurately and precisely with a high dose while geometrically sparing adjacent normal tissues. It enables accomplishing radiotherapy in a week without interrupt of the chemotherapy course. However, the standard dose and volume for SBRT in pancreatic cancer are to be explored.

To rule out the impact of radiotherapy on the site of recurrence, only those patients who did not receive adjuvant radiotherapy were included in this study. We map the postoperative local recurrences of pancreatic cancer patients with respect to major arteries in radiographic imaging to explore the areas at the highest risk for local recurrence, and then to provide a suitable target volume for adjuvant radiotherapy of pancreatic cancer, and to achieve dose escalation. This may allow decreased treatment-related toxicity and increased probability of disease control.

\section{Methods and materials}

\section{Patients}

This study was approved by the Institutional Review Board of the Second Affiliated Hospital, Zhejiang University School of Medicine (SAHZU). From January 2010 to August 2015, 305 patients had undergone surgery for the primary pancreatic cancer at SAHZU, histopathology diagnosis were achieved after surgery. The medical records were retrospectively reviewed. Patients were included if they met the following inclusion criteria: 1) patients after surgery for the primary tumor in pancreas; 2) patients with local recurrence, with or without distant metastasis; 3) patients did not receive neoadjuvant or adjuvant radiation. On the other hand, patients were excluded based on the following: 1) patients after Palliative surgery; 2) patients had no follow-up abdominal Computed Tomography (CT) or Magnetic Resonance Imaging (MRI) in our institution; 3) cases without local recurrence. Local recurrence was defined as progression of soft tissue at the resection area or surrounding the peripancreatic vessels and progression of retroperitoneal lymph nodes in follow-up imaging. A radiologist specializing in abdominal neoplasms imaging identified the recurrences. Rising of CA199 and deterioration of patients' symptom and physical condition were also helpful. Multidisciplinairy team was attended for uncertain case. Patients were classified into three groups: pancreatic head cancer (PHC), pancreatic body cancer (PBC) and pancreatic tail cancer (PTC). In each group, patients were categorized into subgroups based on adjuvant therapy they received: adjuvant chemotherapy alone (CTA) or no adjuvant therapy (NA). The following data were collected for each local recurrent patient: age, sex, tumor diameter, $\mathrm{T}$ stage, $\mathrm{N}$ stage, resection margins (R0: grossly complete resection with microscopically negative margins; $\mathrm{R} 1$ : tumor invasion within $1 \mathrm{~mm}$ from the resection margins; R2: grossly incomplete resection), histological type, comprehensive treatment pattern, type of recurrence and resection type.

\section{Three-dimensional local recurrence map}

All local recurrences were plotted on the CT image of a representative patient according to their relative coordinates to superior mesenteric artery (SMA) or celiac axis (CA) to construct a three-dimensional local recurrence map, which was produced by Raystation software 
Table 1 Baseline clinical characteristics

\begin{tabular}{|c|c|c|c|c|c|c|c|}
\hline Characteristic & $\begin{array}{l}\text { All(\%) } \\
n=83\end{array}$ & $\begin{array}{l}\mathrm{PHC}(\%) \\
n=62\end{array}$ & $\begin{array}{l}\mathrm{PBC}(\%) \\
n=13\end{array}$ & $\begin{array}{l}\text { PTC(\%) } \\
n=8\end{array}$ & $\begin{array}{l}\text { PHC vs PBC } \\
p \text { value }\end{array}$ & $\begin{array}{l}\text { PBC vs PTC } \\
p \text { value }\end{array}$ & $\begin{array}{l}\text { PHC vs PTC } \\
p \text { value }\end{array}$ \\
\hline Age, mean(year) & 62 & 62 & 62 & 65 & 0.951 & 0.328 & 0.336 \\
\hline rang & $36-81$ & $36-81$ & $50-70$ & $47-75$ & & & \\
\hline \multicolumn{8}{|l|}{ Sex } \\
\hline Male & $51(61)$ & $37(60)$ & $8(62)$ & $6(75)$ & & & \\
\hline Female & $32(39)$ & $25(40)$ & $5(38)$ & $2(25)$ & 0.901 & 0.656 & 0.651 \\
\hline Tumor diameter, mean(mm) & 34.8 & 34.4 & 34.2 & 39.3 & 0.963 & 0.32 & 0.261 \\
\hline range & $17.5-80$ & $17.5-80$ & $21-55$ & $25-65$ & & & \\
\hline \multicolumn{8}{|l|}{ T stage } \\
\hline $\mathrm{T} 2$ & $12(14)$ & $7(11)$ & $4(31)$ & $1(13)$ & & & \\
\hline T3 & $53(64)$ & $41(66)$ & $5(38)$ & $7(88)$ & & & \\
\hline T4 & $18(22)$ & $14(23)$ & $4(31)$ & $0(0)$ & 0.08 & 0.104 & 0.358 \\
\hline \multicolumn{8}{|l|}{ N stage } \\
\hline NO & $45(54)$ & $31(50)$ & $8(62)$ & $6(75)$ & & & \\
\hline N1 & $38(46)$ & $31(50)$ & $5(38)$ & $2(25)$ & 0.449 & 0.656 & 0.339 \\
\hline \multicolumn{8}{|l|}{ Resection marging } \\
\hline RO & $80(96)$ & $59(95)$ & 13(100) & $8(100)$ & & & \\
\hline R1 & $3(4)$ & $3(5)$ & $0(0)$ & $0(0)$ & 0.755 & & 1 \\
\hline \multicolumn{8}{|l|}{ Histological type } \\
\hline Ductal adenocarcinoma & $76(92)$ & $59(95)$ & $11(85)$ & $6(75)$ & & & \\
\hline Intraductal papillary-mucinous tumor & $2(2)$ & 1(2) & $0(0)$ & $1(13)$ & & & \\
\hline Mucoepidermoid carcinoma & $3(4)$ & $1(2)$ & $1(8)$ & $1(13)$ & & & \\
\hline Neuroendocrine neoplasms & $2(2)$ & $1(2)$ & $1(8)$ & $0(0)$ & 0.205 & 0.809 & 0.097 \\
\hline \multicolumn{8}{|l|}{ Comprehensive treatment pattern } \\
\hline no adjuvant therapy & $31(37)$ & 24(39) & $3(23)$ & $4(50)$ & & & \\
\hline adjuvant chemotherapy alone & $52(63)$ & $38(61)$ & $10(77)$ & $4(50)$ & 0.453 & 0.346 & 0.818 \\
\hline \multicolumn{8}{|l|}{ Resection type } \\
\hline pancreatoduodenectomy & $53(64)$ & $53(85)$ & $0(0)$ & $0(0)$ & & & \\
\hline distal pancreatectomy & $21(25)$ & $0(0)$ & 13(100) & $8(100)$ & & & \\
\hline pancreatoduodenectomy \& total pancreatectomy & $7(8)$ & $7(11)$ & $0(0)$ & $0(0)$ & & & \\
\hline pancreatoduodenectomy \& distal pancreatectomy & $2(2)$ & 2(3) & $0(0)$ & $0(0)$ & $<0.001$ & & $<0.001$ \\
\hline \multicolumn{8}{|l|}{ Type of recurrence } \\
\hline Local only & $31(37)$ & $25(40)$ & $4(31)$ & $2(25)$ & & & \\
\hline local \& metastasis & $52(63)$ & $37(60)$ & $9(69)$ & 6(75) & 0.52 & 1 & 0.651 \\
\hline Relapse-free time interval, mean(month) & 8.4 & 8.4 & 6.8 & 11.1 & 0.507 & 0.253 & 0.38 \\
\hline range & $1.1-43.2$ & $1.1-43.2$ & $1.4-15.5$ & $1.3-39.8$ & & & \\
\hline
\end{tabular}

Abbreviations: PHC pancreatic head cancer, PBC pancreatic body cancer, PTC pancreatic tail cancer

(Raysearch, Stockholm, Sweden). The three-dimensional location of the center of recurrent tumor relative to the center of the SMA or CA of each patient with local failure was measured. To choose SMA or CA depended on which artery was closer to the tumor. SMA and CA were contoured from the origin of the arteries to $30 \mathrm{~mm}$ and $10 \mathrm{~mm}$ along the natural anatomy of the arteries inferiorly, respectively. The center of the tumor and the center of the arteries were located by Raystation software as mentioned above.

\section{Novel adjuvant field delineation}

A radiation target volume for adjuvant radiotherapy was designed to the areas where local failures commonly occurred according to the three-dimensional local recurrence map. The center of all plotted recurrences 


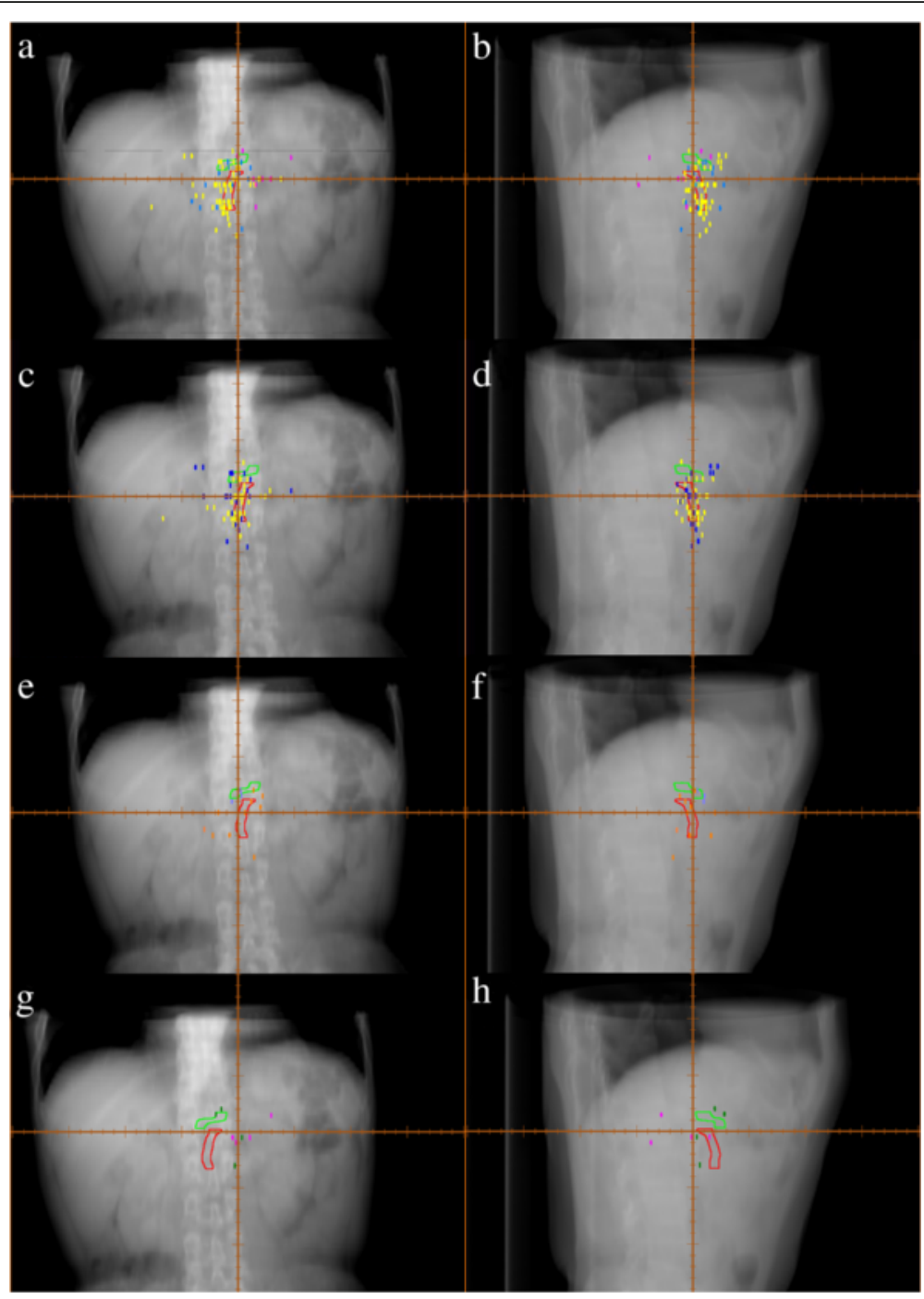

Fig. 1 Digitally reconstructed radiograph of local recurrences. a Anterior-posterior and $\mathbf{b}$ lateral views of local recurrence plots with respect to the superior mesenteric artery (red) and celiac artery (green) for postoperative patients with pancreatic head(yellow), body(blue), and tail (peach) cancer. c Anterior-posterior and $\mathbf{d}$ lateral views of local recurrence plots with respect to the superior mesenteric artery (red) and celiac artery (green) for postoperative patients with chemotherapy alone (yellow) or no adjuvant therapy (blue) in pancreatic head cancer. e Anterior-posterior and $\mathbf{f}$ lateral views of local recurrence plots with respect to the superior mesenteric artery (red) and celiac artery (green) for postoperative patients with chemotherapy alone (orange) or no adjuvant therapy (lavender) in pancreatic head cancer. $\mathbf{g}$ Anterior-posterior and $\mathbf{h}$ lateral views of local recurrence plots with respect to the superior mesenteric artery (red) and celiac artery (green) for postoperative patients with chemotherapy alone (peach) or no adjuvant therapy (dark green) in pancreatic head cancer

was located by Raystation software which had been mentioned above. Then $90 \%$ and $80 \%$ of plotted recurrences closer to the center were produced respectively. A combined contour structure of the SMA and CA was expanded to cover $90 \%$ and $80 \%$ plotted recurrences, thus to generate clinical target volume (CTV)-90 and CTV-80. Planning target volume (PTV)-90 and PTV-80 was created through expanding CTV-90 and CTV-80 by $3 \mathrm{~mm}$, respectively.

\section{Treatment planning}

We applied the proposed plan in four simulated patients. Organs at risk (OARs) included the liver, stomach, small intestine, large bowel, spinal cord and kidneys. Doses constraints of OARs were based on a study of Herman et al. [13]. PTV-90 and PTV-80 were retracted to avoid the stomach, small intestine, and large bowel by $3 \mathrm{~mm}$ to generate PTV-90_modified and PTV-80_modified. For the adjuvant SBRT treatment plans, 25 Gy in 5-Gy fractions was delivered to PTV-90_modified, 33 Gy in 6.6 Gy fractions was delivered to PTV-80_modified as simultaneous integrated boost. More than $90 \%$ of each target volume received $100 \%$ of the prescription dose, and no more than $1 \mathrm{cc}$ of PTV-80_modified received more than $120 \%$ of the prescription dose. The plans 
Table 2 Comparison of baseline clinical characteristics between NA and CTA groups in pancreatic head cancer

\begin{tabular}{|c|c|c|c|}
\hline Characteristic & $\begin{array}{l}\mathrm{NA}(\%) \\
n=24\end{array}$ & $\begin{array}{l}\text { CTA(\%) } \\
n=38\end{array}$ & $\begin{array}{l}\text { NA vs CTA } \\
p \text { value }\end{array}$ \\
\hline Age, mean(year) & 65 & 60 & 0.038 \\
\hline rang & $45-81$ & $36-74$ & \\
\hline \multicolumn{4}{|l|}{ Sex } \\
\hline Male & $13(54)$ & $24(63)$ & \\
\hline Female & $11(46)$ & $14(37)$ & 0.482 \\
\hline Tumor diameter, mean(mm) & 34.1 & 34.5 & 0.884 \\
\hline range & $20-57.5$ & $17.5-80$ & \\
\hline \multicolumn{4}{|l|}{ T stage } \\
\hline $\mathrm{T} 2$ & $3(13)$ & $4(11)$ & \\
\hline T3 & $14(58)$ & $27(71)$ & \\
\hline T4 & $7(29)$ & $7(18)$ & 0.532 \\
\hline \multicolumn{4}{|l|}{$N$ stage } \\
\hline No & $12(50)$ & 19(50) & \\
\hline N1 & $12(50)$ & 19(50) & 1 \\
\hline \multicolumn{4}{|l|}{ Resection marging } \\
\hline RO & $24(100)$ & $35(92)$ & \\
\hline R1 & $0(0)$ & $3(8)$ & 0.422 \\
\hline \multicolumn{4}{|l|}{ histological type } \\
\hline Dactal adenocarcinoma & $22(92)$ & $37(97)$ & \\
\hline Intraductal papillary-mucinous tumor & $1(4)$ & $0(0)$ & \\
\hline Mucoepidermoid carcinoma & $0(0)$ & 1(3) & \\
\hline Neuroendocrine neoplasms & $1(4)$ & $0(0)$ & 0.331 \\
\hline \multicolumn{4}{|l|}{ Resection type } \\
\hline pancreatoduodenectomy & 19(79) & $35(92)$ & \\
\hline distal pancreatectomy & $0(0)$ & $0(0)$ & \\
\hline pancreatoduodenectomy \& total pancreatectomy & $4(17)$ & $2(5)$ & \\
\hline pancreatoduodenectomy \& distal pancreatectomy & $1(4)$ & $1(3)$ & 0.315 \\
\hline \multicolumn{4}{|l|}{ Type of recurrence } \\
\hline Local only & $7(29)$ & $18(47)$ & \\
\hline local \& metastasis & $17(71)$ & 20(53) & 0.155 \\
\hline Relapse-free time interval, mean(month) & 7.9 & 8.7 & 0.644 \\
\hline range & $1.1-43.2$ & $1.4-17.8$ & \\
\hline
\end{tabular}

Abbreviations: NA no adjuvant therapy, CTA adjuvant chemotherapy alone

were delivered in 5 consecutive days. They were not applied in real patients.

A standard radiation plan based on RTOG 0848 [14] was also employed in these four cases. Regions of interest (ROIs) included most proximal $10 \mathrm{~mm}$ of the CA and most proximal $30 \mathrm{~mm}$ of the SMA from the take-off from the aorta, portal vein(PV), preoperative tumor volume, pancreaticojejunostomy (PJ) and aorta from the most cephalad of CA, PJ or PV to the bottom of the second lumbar vertebra. Then CA, SMA and PV were expanded by $10 \mathrm{~mm}$ in all directions. PJ was expanded by $5-10 \mathrm{~mm}$ in all directions. The preoperative tumor volume was expanded by $5-10 \mathrm{~mm}$ in all directions. Aorta was expanded by $25 \mathrm{~mm}$ to the right, $10 \mathrm{~mm}$ to the left, $20-25 \mathrm{~mm}$ to the anterior, $2 \mathrm{~mm}$ to the posterior and $0 \mathrm{~mm}$ to the inferior. The expansion of aorta in the superior direction should up to the most superior slice of CA, PJ or PV expansion. The CTV was created by merging the above ROI expansions. Normal structures such as liver, stomach, small intestine, large bowel, left kidney, right kidney and spinal canal were contoured. Dose-volume constraints also followed RTOG 0848 guideline [14]. The CTV was edited to be adjacent to a dose limited normal organ if the noted expansions 


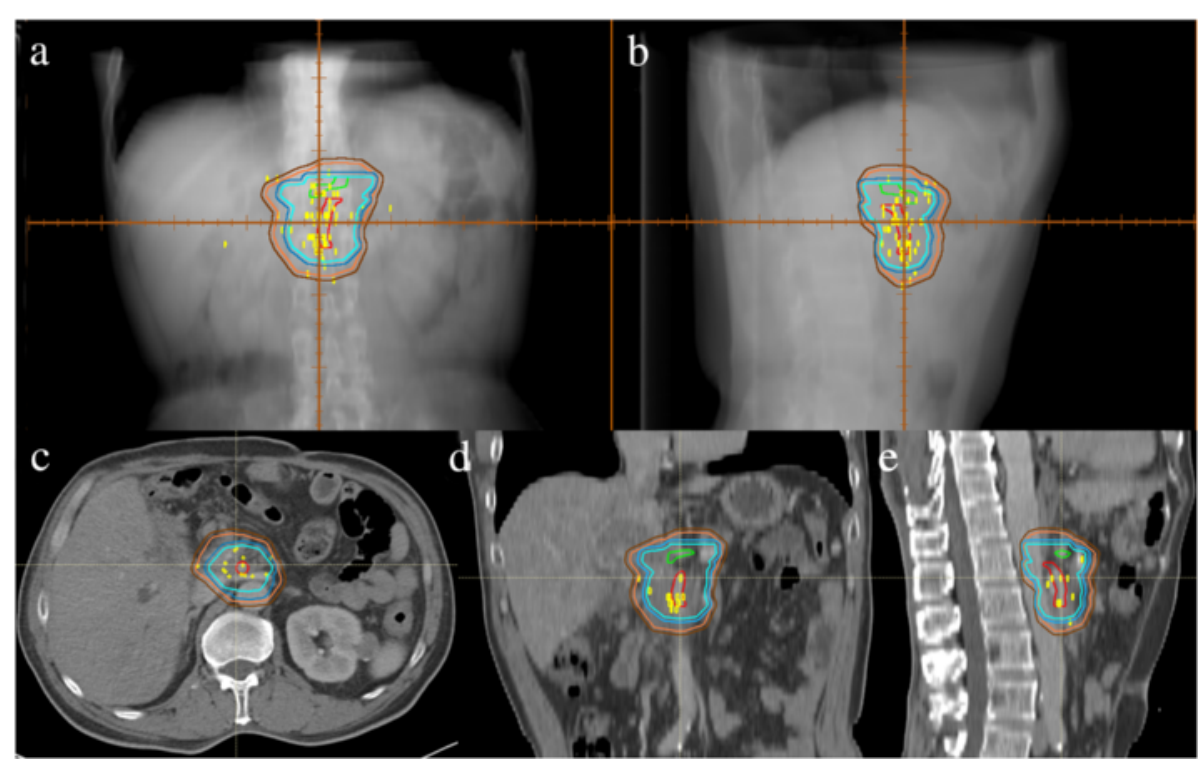

Fig. 2 CTV-90 (orange) and CTV-80 (light blue) which target the regions where $90 \%$ and $80 \%$ of recurrences occur and their corresponding PTV-90 (brown) and PTV-80 (dark blue) in one simulated patient with pancreatic head cancer in anterior-posterior (a), lateral (b), axial (c), coronal (d) and sagittal (e) views

protruded into it to construct CTV_modified. The PTV_modified was created through expanding the CTV by $5 \mathrm{~mm}$ in all directions. PTV_modified was delivered 50.4 Gy in 1.8-Gy fractions in 28 consecutive daily fractions over 5 to 6 weeks. More than $90 \%$ of the target volume received $100 \%$ of the prescription dose.

\section{Statistical analysis}

Normality test (Kolmogorov-Smirnov test) and homogeneity of variance test (Levene test) were conducted in measurement data such as age, tumor diameter and Relapse-free time interval among subgroups. In each test, $\mathrm{P}>0.1$ was considered Gaussian distribution and homogeneity of variance, respectively. Two-group $t$-test was conducted among subgroups to compare the measurement data if they were Gaussian distribution and homogeneity of variance. Chi-square test and Fisher's exact test was conducted among subgroups to compare enumeration data such as $\mathrm{T}$ stage, $\mathrm{N}$ stage, resection margin, histological type, comprehensive treatment pattern, resection type and type of recurrence. $\mathrm{P}<0.05$ was considered significant. IBM SPSS Statistics 19.0 was used for statistical analyses. Raystation v4.5.1.14 was used for analyzing high throughput physics data.

\section{Results}

Patient clinical characteristics

Of the 305 patients, 83 patients met the criteria were included in the study. Among them, 62 had PHC, 13 had PBC and 8 had PTC. Table 1 shows the baseline clinical characteristics. The measurement data such as age, tumor diameter and Relapse-free time interval in each subgroup were Gaussian distribution and homogeneity of variance. The mean recurrence-free time interval was 8.4 months (range, 1.1-43.2 month), specifically 8.4 month (range, 1.1-43.2 months), 6.8 months (range, 1.4-15.5 months), 11.1 months (range, 1.339.8 months) for PHC, PBC, PTC respectively. Forty five (54\%) patients was in stage N0 and 38 (46\%) was in stage N1. Sixty two $(75 \%)$ patients undergone pancreatoduodenectomy and twenty one $(25 \%)$ patients undergone distal pancreatectomy. Thirty-one (37 \%) patients undergone NA, 52 (63 \%) patients undergone CTA. Thirty-one $(37 \%)$ patients undergone local recurrence only. The majority of patients with PHC undergone pancreatoduodenectomy, while all patients in $\mathrm{PBC}$ and PTC undergone distal pancreatectomy ( $\mathrm{PHC}$ vs $\mathrm{PBC}$ : $\mathrm{p}<0.001$, PHC vs PTC: $\mathrm{p}<0.001)$. All other baseline clinical characteristics were comparable among patients with PHC, PBC and PTC.

\section{Three-dimensional local recurrence map}

Patterns of local failure by location of pancreatic cancer are shown in Fig. 1. Most recurrences occurred near CA or SMA. Sixty four patients (77 \%) suffered from recurrence closer to SMA, with a mean distance from the center of SMA of $19.5 \mathrm{~mm}, 25 \mathrm{~mm}$ and $36.4 \mathrm{~mm}$ in PHC, PBC and PTC groups respectively. Nineteen patients $(23 \%)$ suffered from recurrence closer to CA, with a mean distance from the center of CA of $18.6 \mathrm{~mm}$, $12.2 \mathrm{~mm}$ and $40.5 \mathrm{~mm}$ in $\mathrm{PHC}, \mathrm{PBC}$ and PTC groups respectively. According to the picture and data, the 
Table 3 Dosimetric parameters of organ of risk for the proposed plan and RTOG 0848 plan in 4 simulated cases

\begin{tabular}{|c|c|c|c|c|c|c|c|c|c|c|}
\hline & \multicolumn{5}{|c|}{ The proposed plan } & \multicolumn{5}{|l|}{ RTOG 0848} \\
\hline & Constraints & Case 1 & Case 2 & Case 3 & Case 4 & Constraints & Case 1 & Case 2 & Case 3 & Case 4 \\
\hline left kidney & & & & & & $\mathrm{V} 18<65 \%$ & $34.12 \%$ & $46.95 \%$ & $47.47 \%$ & $42.25 \%$ \\
\hline right kidney & & & & & & $\vee 18<50 \%$ & $42.27 \%$ & $42.85 \%$ & $37.06 \%$ & $35.36 \%$ \\
\hline \multirow[t]{3}{*}{ Stomach } & $\mathrm{V} 15<9 \mathrm{cc}$ & 7.52 cc & $4.41 \mathrm{cc}$ & $7.65 c c$ & $0.00 c c$ & Dmax $\leq 58$ Gy & 52.72Gy & 52.40Gy & 52.79Gy & 34.55Gy \\
\hline & $\mathrm{V} 20<3 \mathrm{cc}$ & 1 & $0.26 \mathrm{cc}$ & $0.51 \mathrm{cc}$ & $0.00 \mathrm{cc}$ & V $56<10 \%$ & $0.00 \%$ & $0.00 \%$ & $0.00 \%$ & $0.00 \%$ \\
\hline & & & & & & $V 52<15 \%$ & $0.04 \%$ & $0.07 \%$ & $0.05 \%$ & $0.00 \%$ \\
\hline \multirow[t]{3}{*}{ Small intestine } & $\mathrm{V} 15<9 \mathrm{cc}$ & $8.27 \mathrm{cc}$ & $4.76 \mathrm{cc}$ & $3.24 c c$ & $8.26 c c$ & Dmax $\leq 58 G y$ & 55.67Gy & 53.16Gy & 53.26Gy & 54.08Gy \\
\hline & $\mathrm{V} 20<3 \mathrm{cc}$ & $1.08 c c$ & $1.82 \mathrm{cc}$ & $0.36 c c$ & $1.12 \mathrm{cc}$ & V $56<10 \%$ & $0.00 \%$ & $0.00 \%$ & $0.00 \%$ & $0.00 \%$ \\
\hline & & & & & & V $52<15 \%$ & $8.83 \%$ & $3.42 \%$ & $0.25 \%$ & $2.87 \%$ \\
\hline Liver & $\mathrm{V} 12<50 \%$ & $21.86 \%$ & $14.92 \%$ & $7.99 \%$ & $9.09 \%$ & Dmean $\leq 30 G y$ & 23.46Gy & 16.57Gy & 15.66Gy & 15.86Gy \\
\hline Spinal cord & $\mathrm{V} 8<1 \mathrm{cc}$ & $0.04 c c$ & $0.09 c c$ & $0.01 c c$ & $0.13 c c$ & $\mathrm{~V} 50<0.03 \mathrm{cc}$ & $0.00 \mathrm{cc}$ & $0.00 \mathrm{cc}$ & $0.00 c c$ & $0.00 \mathrm{cc}$ \\
\hline Combined kidney & $\mathrm{V} 12<75 \%$ & $48.27 \%$ & $37.87 \%$ & $36.89 \%$ & $49.68 \%$ & & & & & \\
\hline \multirow[t]{2}{*}{ Large bowel } & $\mathrm{V} 15<9 \mathrm{cc}$ & $6.92 c c$ & $3.22 \mathrm{cc}$ & 6.62 & $6.02 c c$ & & & & & \\
\hline & $\mathrm{V} 20<3 \mathrm{cc}$ & $1.60 \mathrm{cc}$ & $0.00 \mathrm{cc}$ & $0.00 \mathrm{cc}$ & $0.16 \mathrm{cc}$ & & & & & \\
\hline Proximal large bowel & $\mathrm{V} 33<1 \mathrm{CC}$ & $0.00 \mathrm{cc}$ & $0.00 \mathrm{cc}$ & $0.00 \mathrm{cc}$ & $0.00 \mathrm{cc}$ & & & & & \\
\hline Proximal stomach & $\mathrm{V} 33<1 \mathrm{CC}$ & $0.00 \mathrm{cc}$ & $0.00 \mathrm{cc}$ & $0.00 \mathrm{cc}$ & $0.00 \mathrm{cc}$ & & & & & \\
\hline Proximal small intestine & $\mathrm{V} 33<1 \mathrm{cc}$ & $0.00 \mathrm{cc}$ & $0.00 \mathrm{cc}$ & $0.00 \mathrm{cc}$ & $0.00 \mathrm{cc}$ & & & & & \\
\hline
\end{tabular}

Abbreviations: RTOG Radiation Therapy Oncology Group

recurrence pattern of PTC seems at the left-rear of SMA and $\mathrm{CA}$, while the recurrence location of $\mathrm{PHC}$ and $\mathrm{PBC}$ tend to be surrounding SMA and CA.

Patterns of local failure classified by adjuvant therapy patients with $\mathrm{PHC}$ and $\mathrm{PBC}$ received are shown in Fig. 1. In patients with PHC, 24 had NA, 38 had CTA. In patients with PBC, 3 had NA, 10 had CTA. In patients with PHC, $50(81 \%)$ suffered from recurrence closer to SMA, with a mean distance from the center of SMA of $19.4 \mathrm{~mm}$ and $19.9 \mathrm{~mm}$ in the NA and CTA groups respectively. Twelve patients $(23 \%)$ suffered from recurrence closer to CA, with a mean distance from the center of CA of $20.6 \mathrm{~mm}$ and $14.4 \mathrm{~mm}$ in NA and CTA groups respectively. In patients with PBC, 9 (69 \%) suffered from recurrence closer to SMA, with a mean distance from the center of SMA of $21.8 \mathrm{~mm}, 25.4 \mathrm{~mm}$ in the NA, CTA groups respectively. Four patients (31\%) suffered from recurrence closer to CA, with a mean distance from the center of CA of $12.2 \mathrm{~mm}$ and $12.1 \mathrm{~mm}$ in NA and CTA groups respectively.

Table 2 shows the comparison of baseline clinical characteristics between NA and CTA groups in pancreatic head cancer. The NA patients were older, with a median age of 65 years compared with 60 years in the CTA groups $(p=0.038)$. All other baseline clinical characteristics were comparable among patients receiving $\mathrm{NA}$ and CTA.

\section{Production of treatment target volume}

By expanding the combined SMA and CA contour structure, the CTV for SBRT of pancreatic head cancer was constructed to generate a volume that included areas where failure was frequent. Ninety percent of recurrences were contained by a $30 \mathrm{~mm}$ right-lateral, $21 \mathrm{~mm}$ left-lateral, $20 \mathrm{~mm}$ anterior, $13 \mathrm{~mm}$ posterior, $10 \mathrm{~mm}$ superior, and $20 \mathrm{~mm}$ inferior expansion of the combined SMA and CA contours to generate CTV-90. Eighty percent of recurrences were contained by a $20 \mathrm{~mm}$ right-lateral, $18 \mathrm{~mm}$ left-lateral, $12 \mathrm{~mm}$ anterior, $12 \mathrm{~mm}$ posterior, $0 \mathrm{~mm}$ superior, and $10 \mathrm{~mm}$ inferior expansion of the combined SMA and CA contours to create CTV-80. PTV-90 and PTV-80 was created through expanding CTV-90 and CTV- 80 by $3 \mathrm{~mm}$, respectively (Fig. 2).

\section{Comparison of the proposed plan with standard radiation plan}

We applied this plan and the standard radiation plan recommended by RTOG 0848 in four simulated patients. Doses constraints of OARs of the proposed plan and RTOG 0848 are seen in Table 3. PTV-90_modified and PTV-80_modified were generated by adjusting PTV-90 and PTV-80 to avoid the stomach, jejunum, and bowel by $3 \mathrm{~mm}$. For RTOG 0848 plan, CTV_modified was generated by editing CTV to be adjacent to doselimited normal organ if it protrudes into relative structure. PTV_modified was generated through expanding CTV_modified by $5 \mathrm{~mm}$ (Fig. 3). The areas of the proposed PTV-90_modified and PTV-80_modifed were much smaller than RTOG 0848 PTV_modified. Figure 4 illustrates comparison of the proposed plan and RTOG 


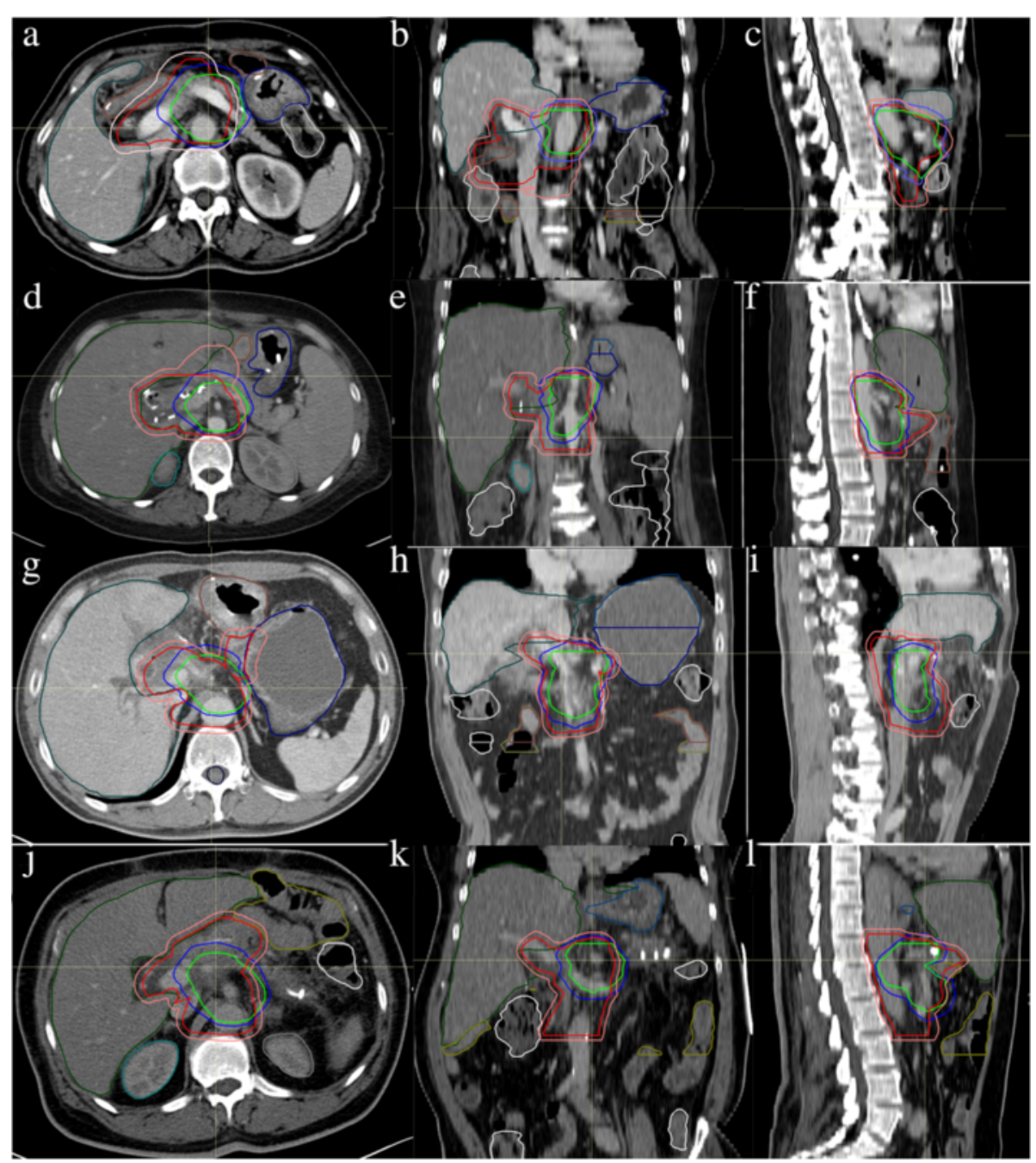

Fig. 3 The proposed PTV-80_modified (green) and PTV-90_modified (blue) of this study are shown simultaneously with RTOG 0848 CTV_modified (red) and PTV_modified (pink) in 4 simulated cases. Axial, coronal and sagittal views are shown for case 1 (a, $\mathbf{b}$, and $\mathbf{c}$ ), case 2 (d), e, and $\mathbf{f})$, case 3 (g, h, and $\mathbf{i}$ ) and case 4 (j, $\mathbf{k}$ and $\mathbf{I}$ )

0848 plan in 4 simulated cases in the same sections. The average volume of PTV-90_modified and PTV-80_modified were $197 \mathrm{cc}$ and $113 \mathrm{cc}$, respectively. However, the average volume of PTV_modified according to RTOG 0848 was $466 \mathrm{cc}$, which was much larger than the target volume in the proposed plan (Table 4). OARs and target volume in all plans reached the dosimetric constraints (Tables 3 and 5). Table 6 compares the dosimetric parameters between the proposed plan and the plan according to RTOG 0848 in the 4 simulated cases. The dose OARs received in the proposed plan was much lower than that in the plan according to RTOG 0848.

\section{Discussion}

So far there were limited studies focusing on recurrence pattern of pancreatic cancer. Herman et al. first demonstrated that a majority of local recurrences in postoperative patients with $\mathrm{PHC}$ are contained within a small region surrounding the $\mathrm{CA}$ and SMA and created an anatomic map with areas at the highest risk for local recurrence [13]. In their data, a majority of patients have undergone radiation. However, radiation therapy may reduce the risk of recurrence within the irradiation volume, thus influence the spatial location of local recurrence. Here, in this study, we followed the study of Herman et al. but only included patients without radiotherapy to rule out the impact of radiotherapy on the recurrence pattern.

In glioma, contouring the target volume according to the recurrence pattern has been proved feasible. Chang et al. have investigated the recurrence pattern in patients with glioblastoma after surgery and chemoradiotherapy. They successfully demonstrated that the smaller target 

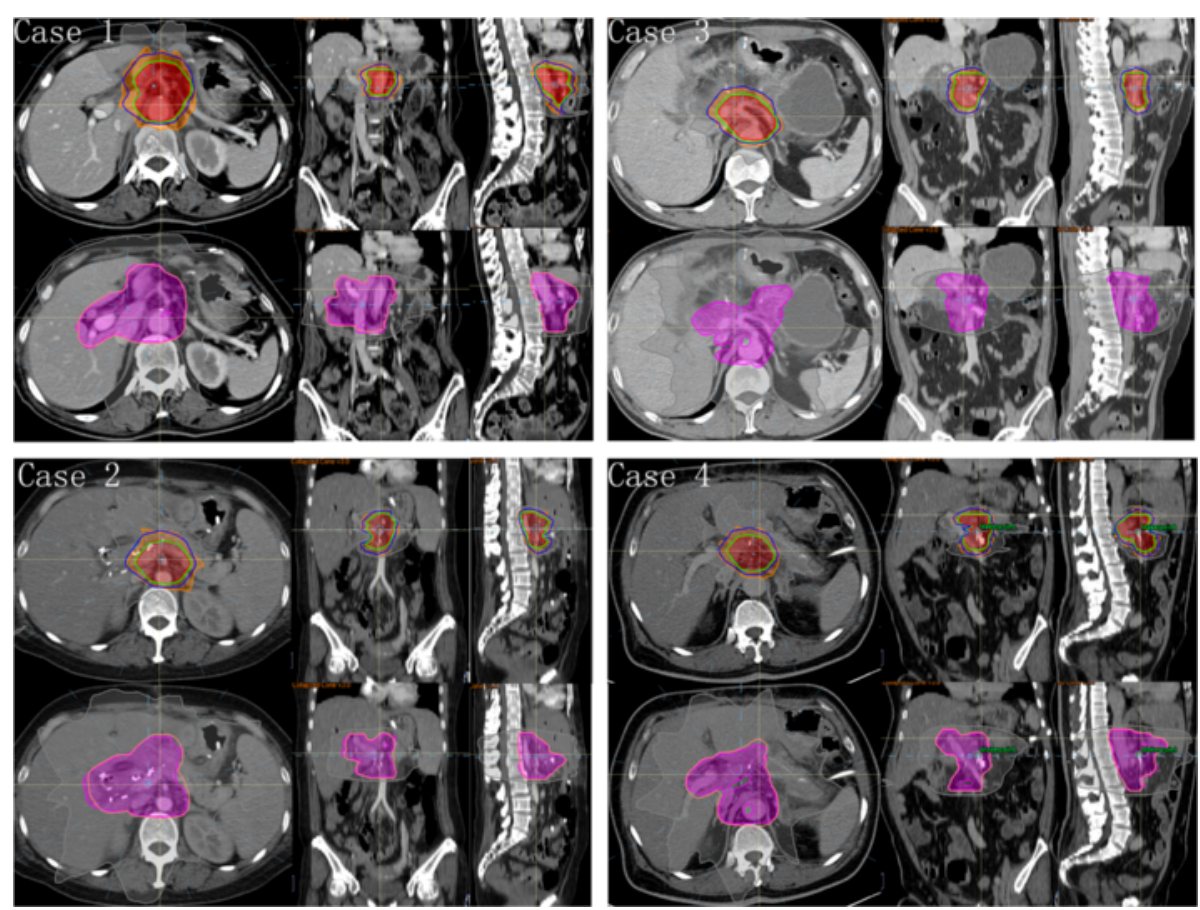

Fig. 4 Comparison of the proposed plan (the first row in each case) and RTOG 0848 plan (the second row in each case) in 4 simulated cases. In each case, Axial, sagittal, and coronal views are shown, which are in the same section in both plans, respectively. PTV-90_modified (blue), PTV80_modified (green), PTV_modified (pink), 33 Gy isodose line (red), 25 Gy isodose line (orange), 50.4 Gy isodose line (peach), 45.45 \% isodose line (grey) are indicated on each plan

volume would not increase the pattern of local failure $[15,16]$. In pancreatic cancer, Heye et al. have reported the recurrence pattern in postoperative patients. They, from the viewpoint of radiologists, found the SMA is the leading structure for recurrence [17]. While in our study, a three-dimensional map of the local recurrences based on their relative coordinates to SMA or CA was further generated to guide target volume contouring for radiotherapy. Figure 1 suggests that the distribution of local recurrences between NA and CTA are similar in pancreatic cancer, and we found that the recurrence pattern have correlation with the location of primary tumor. The recurrence location of PTC seemed at the left of the CA and SMA and was far from them. It seems improper to create an adjuvant treatment volume for PTC based on expansions of the CA and SMA. We didn't do further analysis of $\mathrm{PBC}$ as the patients number of $\mathrm{PBC}$ in our study is small. We then focused on the analysis of pancreatic head cancer. The target volumes were created by expanding the structure of the SMA and CA to cover $90 \%$ and $80 \%$ plotted recurrences (Fig. 2). As shown in Fig. 3, the target volume proposed in this study was much smaller than that in RTOG 0848. The low number of patients ( $\mathrm{n}=4$ cases) used for the comparison to the RTOG protocol might be a limitation factor. However, the volume of PTV-80 was only one-fourth of that of RTOG 0848 PTV (Table 4), these cases exhibit good example. It is reasonable to apply these smaller treatment

Table 4 Target volume in the proposed plan and RTOG0848 plan

\begin{tabular}{llllllll}
\hline & \multicolumn{2}{l}{ PTV(cC) } & & & \multicolumn{2}{l}{ PTV_modified(cc) } \\
\cline { 2 - 3 } & PTV(RTOG0848) & PTV-90 & PTV-80 & & $\begin{array}{l}\text { PTV_modified } \\
\text { (RTOG0848) }\end{array}$ & PTV-90_modified & PTV-80_modified \\
\hline Case 1 & 484.33 & 226.64 & 121.71 & & 444.84 & 191.02 & 110.28 \\
Case 2 & 368.54 & 190.49 & 103.62 & & 356.26 & 166.83 & 94.66 \\
Case 3 & 518.92 & 209.81 & 115.23 & & 506.43 & 205.43 & 114.88 \\
Case 4 & 586.39 & 251.9 & 140.61 & & 556.43 & 224.61 & 130.95 \\
Average & 489.545 & 219.71 & 120.2925 & & 465.99 & 196.9725 & 112.6925 \\
\hline
\end{tabular}

Abbreviations: PTV-90_modified planning target volume covering $90 \%$ plotted recurrences with avoidance of proximal organs at risk, PTV-80_modified planning target volume covering $80 \%$ plotted recurrences with avoidance of proximal organs at risk 
Table 5 Plan characteristics for the proposed plan and RTOG 0848 plan in 4 simulated cases

\begin{tabular}{|c|c|c|c|c|c|}
\hline & Constraints & Case 1 & Case 2 & Case 3 & Case 4 \\
\hline \multirow[t]{2}{*}{ PTV-80_modified } & V39.6<1 cc & $0.72 \mathrm{cc}$ & $0.90 \mathrm{cc}$ & $0.87 \mathrm{cc}$ & $0.47 c c$ \\
\hline & $V 33 \geq 90 \%$ & $90 \%$ & $90 \%$ & $90 \%$ & $90 \%$ \\
\hline PTV-90_modified & V25 $\geq 90 \%$ & $98.64 \%$ & $98.75 \%$ & $93.33 \%$ & $98.81 \%$ \\
\hline PTV_modified (RTOG0848) & $V 50.4 \geq 95 \%$ & $95 \%$ & $95 \%$ & $95 \%$ & $95 \%$ \\
\hline
\end{tabular}

Abbreviations: PTV-90_modified planning target volume covering $90 \%$ plotted recurrences with avoidance of proximal organs at risk, PTV-80_modified planning target volume covering $80 \%$ plotted recurrences with avoidance of proximal organs at risk

volumes in clinical practices because it might reduce radiation related toxicity and achieve dose escalation. The treatment plans according to this study and RTOG 0848 in 4 cases were further generated, which indicated the shrinked areas could potentially reduce the dose to
OARs, then minimize the radiation related toxicity (Fig. 4, Tables 4 and 6).

To our knowledge, this study is the first research in pancreatic cancer which produced a map of local recurrences in postoperative patients who did not

Table 6 Comparison of dosimetric parameters between the proposed plan and RTOG 0848 plan in 4 simulated cases (cGy)

\begin{tabular}{|c|c|c|c|c|c|c|c|c|c|c|c|c|}
\hline & \multicolumn{3}{|c|}{ The proposed plan } & \multicolumn{3}{|c|}{ RTOG 0848} & \multicolumn{3}{|c|}{ The proposed plan } & \multicolumn{3}{|c|}{ RTOG 0848} \\
\hline & D99 & D50 & D1 & D99 & D50 & D1 & D99 & D50 & D1 & D99 & D50 & D1 \\
\hline & Case 1 & & & & & & Case & & & & & \\
\hline PTV_modified (RTOG0848) & & & & 5010 & 5132 & 5303 & & & & 5004 & 5101 & 5243 \\
\hline left kidney & 251 & 1004 & 2234 & 1456 & 1797 & 3080 & 41 & 505 & 2182 & 138 & 1750 & 4625 \\
\hline right kidney & 65 & 374 & 1601 & 578 & 1527 & 5299 & 40 & 392 & 2241 & 123 & 1477 & 4749 \\
\hline Stomach & 92 & 676 & 1956 & 293 & 1898 & 4392 & 69 & 599 & 1654 & 225 & 2123 & 4865 \\
\hline Small intestine & 66 & 699 & 1904 & 360 & 2801 & 5267 & 104 & 739 & 1614 & 382 & 3238 & 5147 \\
\hline Liver & 29 & 526 & 3211 & 117 & 2477 & 5209 & 11 & 270 & 2737 & 75 & 1493 & 5137 \\
\hline Spinal cord & 2 & 131 & 702 & 9 & 675 & 4564 & 2 & 72 & 697 & 1 & 275 & 3728 \\
\hline Large bowel & 0 & 3 & 1420 & 0 & 9 & 5169 & 0 & 3 & 1246 & 0 & 22 & 2658 \\
\hline Proximal large bowel & 74 & 628 & 1879 & 458 & 2166 & 5266 & 72 & 284 & 1739 & 260 & 1030 & 3845 \\
\hline Proximal stomach & 104 & 688 & 1959 & 339 & 1915 & 4405 & 99 & 662 & 1671 & 389 & 2247 & 4850 \\
\hline Proximal small intestine & 86 & 847 & 1937 & 643 & 3021 & 5276 & 104 & 739 & 1614 & 368 & 3025 & 5243 \\
\hline PTV-80_modified & 2989 & 3571 & 3947 & & & & 2978 & 3581 & 3934 & & & \\
\hline \multirow[t]{2}{*}{ PTV-90_modified } & 2461 & 3362 & 3923 & & & & 2457 & 3350 & 3904 & & & \\
\hline & Case 3 & & & & & & Case & & & & & \\
\hline PTV_modified (RTOG0848) & & & & 5000 & 5132 & 5230 & & & & 4995 & 5136 & 5249 \\
\hline left kidney & 57 & 654 & 1629 & 271 & 1834 & 3618 & 90 & 836 & 1975 & 1312 & 1904 & 3821 \\
\hline right kidney & 76 & 823 & 1904 & 404 & 1682 & 4443 & 68 & 631 & 1890 & 916 & 1717 & 4036 \\
\hline Stomach & 19 & 128 & 1481 & 104 & 1108 & 5100 & 52 & 134 & 942 & 162 & 510 & 3197 \\
\hline Small intestine & 37 & 500 & 1570 & 273 & 1874 & 5140 & 30 & 379 & 1537 & 364 & 1919 & 5206 \\
\hline Liver & 19 & 222 & 1801 & 118 & 1576 & 5108 & 11 & 230 & 2116 & 103 & 1436 & 5172 \\
\hline Spinal cord & 2 & 96 & 752 & 21 & 868 & 3624 & 1 & 67 & 738 & 4 & 485 & 3602 \\
\hline Large bowel & 0 & 192 & 1500 & 0 & 1064 & 5009 & 0 & 5 & 1531 & 0 & 89 & 4366 \\
\hline Proximal large bowel & 83 & 747 & 1582 & 566 & 2083 & 5052 & 62 & 583 & 1746 & 697 & 2400 & 4807 \\
\hline Proximal stomach & 81 & 490 & 1600 & 733 & 2571 & 5112 & 110 & 394 & 1003 & 492 & 1699 & 3077 \\
\hline Proximal small intestine & 70 & 793 & 1634 & 480 & 2235 & 5187 & 82 & 621 & 1716 & 591 & 2105 & 5257 \\
\hline PTV-80_modified & 3110 & 3596 & 3949 & & & & 2990 & 3557 & 3940 & & & \\
\hline PTV-90_modified & 2380 & 3344 & 3924 & & & & 2478 & 3350 & 3922 & & & \\
\hline
\end{tabular}

Abbreviations: D99 the dose $99 \%$ of the volume received, D50 the dose $50 \%$ of the volume received, D1 the dose $1 \%$ of the volume received, PTV-90 modified planning target volume covering $90 \%$ plotted recurrences with avoidance of proximal organs at risk, PTV-80_modified planning target volume covering $80 \%$ plotted recurrences with avoidance of proximal organs at risk 
receive radiotherapy. It has been proved to be safe in rectal cancer to deliver 25Gy in 5-Gy fractions to the pelvic cavity which contains OARs such as small intestine and large bowel $[18,19]$. In our study, PTV90 modified was delivered a similar schedule as that in rectal cancer. PTV-80_modified was delivered higher dose (33 Gy in 6.6-Gy fractions) which was supported by clinical experience in other gastrointestinal malignancies. The BED of PTV-80_modified was 54.78Gy, which was similar to that of PTV (59.47 Gy) in RTOG 0848. OARs such as small intestine, the average dose $50 \%$ of the volume received (D50) in the 4 cases was 5.79 Gy, while it was much higher in RTOG 0848 plan (24.73 Gy). Large bowel, stomach and other OARs also had lower average D50 than that in RTOG 0848 plan (Table 6). Therefore, with the proposed plan, higher dose to the target volume and lower radiation related toxicity to OARs is achievable.

Up to now, the areas already identified at risk of local recurrence in RTOG 0848 guidelines (aorta, portal vein, tumor bed, and pancreaticojejunostomy) are irradiated at standard doses in clinical practice. In this study, we demonstrated the feasibility of the smaller target volume for radiotherapy in patients with past-operative pancreatic head cancer. However, some limitations still existed. Firstly, recurrence was confirmed by follow-up CT or MRI, but not biopsy which was rarely used in clinical practice at this situation. Although we combined CA199, patients' symptom, physical condition and Multidisciplinairy team for help, imaging diagnosis is still not golden standard. Secondly, the study was based on a static CT, the respiration- and peristalsis-induced shift in OARs was not taken into account. In daily clinical practice, 4D-CT should be utilized to eliminate this factor on dose distribution. Thirdly, we aim to from the viewpoint of evidence-based medicine to explore the target volume of adjuvant radiotherapy of pancreatic cancer, while the study size was not large enough. Fourthly, this study was retrospective analyses on historical cohort, which limited the conclusions. Therefore, there is a need to include more cases and to conduct larger-sample prospective studies on the pattern of recurrence of patients with pancreatic cancer after surgery in the future.

\section{Conclusions}

We produce a map of local recurrence in postoperative patients with pancreatic head cancer. The areas at highest risk for local recurrence are much smaller than the standard adjuvant radiation target volumes based on RTOG consensus guidelines. This might provide reference for adjuvant radiotherapy in patients with pancreatic head cancer to achieve dose escalation and minimize radiation related toxicity.

\section{Abbreviations}

CA: Celiac axis; CRT: Chemoradiation; CT: Abdominal Computed Tomography; CTA: Adjuvant chemotherapy alone; CTV: Clinical target volume;

DRR: Digitally reconstructed radiograph; MRI: Magnetic Resonance Imaging; NA: No adjuvant therapy; NCCR: National Central Cancer Registry; OARs: Organs at risk; Ols: Regions of interest; PBC: Pancreatic body cance; PCA: Pancreatic cancer; PHC: Pancreatic head cancer;

PJ: Pancreaticojejunostomy; PTC: Pancreatic tail cancer; PTV: Planning target volume; PV: Portal vein; RTOG: Radiation Therapy Oncology Group;

SAHZU: The Second Affiliated Hospital, Zhejiang University School of Medicine; SBRT: Stereotactic body radiation therapy; SMA: Superior mesenteric artery

\section{Acknowledgements}

We acknowledge the medical records room of SAHZU for providing the valuable medical records.

\section{Funding}

This study was funded by the National Natural Science Foundation of China (contracts 81572952), and Key Innovative Team for the Diagnosis and Treatment of Pancreatic Cancer of Zhejiang Province (2013TD06).

\section{Availability of data and materials}

Datasets can be retrieved from authors by formal request from interested readers. Datasets will not be directly shared on public link as the national personal data protection act.

\section{Authors' contributions}

WY participated in the design of the study, carried out the clinical data analysis, constructed the three-dimensional image of local recurrences and wrote the manuscript; WH, YS and XR interpreted the clinical data; XZ and $C L$ handled with the physics data. RY and LS read the $C T$ and MRI image. $\mathrm{XB}$ and TL contribute with the clinical data; LZ participated in the design of the study and discussion of the writing; and QW conceived the study, contoured the target volume and wrote the manuscript. All authors read and approved the final manuscript.

\section{Competing interests}

The authors declare that they have no competing interests.

\section{Consent for publication}

Not applicable.

\section{Ethics approval and consent to participate}

This study was approved by the Institutional Review Board of SAHZU. All patients have signed consent to the use of their data for research.

\section{Author details}

${ }^{1}$ Department of Radiation Oncology, the Second Affiliated Hospital, Zhejiang University School of Medicine, Hangzhou, Zhejiang 310009, People's Republic of China. ${ }^{2}$ Department of Hepatobiliary and Pancreatic Surgery, the Second Affiliated Hospital, Zhejiang University School of Medicine, Hangzhou, Zhejiang 310009, People's Republic of China. ${ }^{3}$ Department of Radiology, the Second Affiliated Hospital, Zhejiang University School of Medicine, Hangzhou, Zhejiang 310009, People's Republic of China. ${ }^{4}$ The Sidney Kimmel Comprehensive Cancer Center, the Johns Hopkins University School of Medicine, Baltimore, MD, USA. ${ }^{5}$ Department of Radiation Oncology, the Second Affiliated Hospital, Ministry of Education Key Laboratory of Cancer Prevention and Intervention, Zhejiang University School of Medicine, Jiefang Road 88, Hangzhou 310009, People's Republic of China.

Received: 10 June 2016 Accepted: 13 October 2016

Published online: 19 October 2016

\section{References}

1. Siegel RL, Miller KD, Jemal A. Cancer statistics, 2015. CA Cancer J Clin. 2015;65(1):5-29.

2. Chen W, Zheng R, Baade PD, et al. Cancer statistics in China, 2015. CA Cancer J Clin. 2016;66(2):115-32.

3. Smeenk $\mathrm{HG}$, van Eijck $\mathrm{CH}$, Hop WC, et al. Long-term survival and metastatic pattern of pancreatic and periampullary cancer after adjuvant 
chemoradiation or observation: long-term results of EORTC trial 40891. Ann Surg. 2007;246(5):734-40.

4. Tepper J, Nardi G, Sutt H. Carcinoma of the pancreas: review of MGH experience from 1963 to 1973. Analysis of surgical failure and implications for radiation therapy. Cancer. 1976;37(3):1519-24.

5. Griffin JF, Smalley SR, Jewell W, et al. Patterns of failure after curative resection of pancreatic carcinoma. Cancer. 1990;66(1):56-61.

6. lacobuzio-Donahue CA, Fu B, Yachida S, et al. DPC4 gene status of the primary carcinoma correlates with patterns of failure in patients with pancreatic cancer. J Clin Oncol. 2009;27(11):1806-13.

7. Herman JM, Swartz MJ, Hsu CC, et al. Analysis of fluorouracil-based adjuvant chemotherapy and radiation after pancreaticoduodenectomy for ductal adenocarcinoma of the pancreas: results of a large, prospectively collected database at the Johns Hopkins Hospital. J Clin Oncol. 2008;26(21):3503-10.

8. Klinkenbiil JH, Jeekel J, Sahmoud T, et al. Adjuvant radiotherapy and 5-fluorouracil after curative resection of cancer of the pancreas and periampullary region: phase III trial of the EORTC gastrointestinal tract cancer cooperative group. Ann Surg. 1999;230(6):776-82. discussion 782-774.

9. Neoptolemos JP, Stocken DD, Friess $\mathrm{H}$, et al. A randomized trial of chemoradiotherapy and chemotherapy after resection of pancreatic cancer. N Engl J Med. 2004;350(12):1200-10.

10. Abrams RA, Winter KA, Regine WF, et al. Failure to adhere to protocol specified radiation therapy guidelines was associated with decreased survival in RTOG 9704-a phase III trial of adjuvant chemotherapy and chemoradiotherapy for patients with resected adenocarcinoma of the pancreas. Int J Radiat Oncol Biol Phys. 2012;82(2):809-16.

11. Shah AP, Strauss JB, Abrams RA. Review and commentary on the role of radiation therapy in the adjuvant management of pancreatic cancer. Am J Clin Oncol. 2010;33(1):101-6.

12. Corsini MM, Miller RC, Haddock MG, et al. Adjuvant radiotherapy and chemotherapy for pancreatic carcinoma: the Mayo Clinic experience (1975-2005) J Clin Oncol. 2008;26(21):3511-6.

13. Dholakia AS, Kumar R, Raman SP, et al. Mapping patterns of local recurrence after pancreaticoduodenectomy for pancreatic adenocarcinoma: a new approach to adjuvant radiation field design. Int J Radiat Oncol Biol Phys. 2013;87(5):1007-15.

14. Goodman KA, Regine WF, Dawson LA, et al. Radiation Therapy Oncology Group consensus panel guidelines for the delineation of the clinical target volume in the postoperative treatment of pancreatic head cancer. Int J Radiat Oncol Biol Phys. 2012;83(3):901-8.

15. Chang EL, Akyurek S, Avalos T, et al. Evaluation of peritumoral edema in the delineation of radiotherapy clinical target volumes for glioblastoma. Int J Radiat Oncol Biol Phys. 2007;68(1):144-50.

16. Minniti G, Amelio D, Amichetti $M$, et al. Patterns of failure and comparison of different target volume delineations in patients with glioblastoma treated with conformal radiotherapy plus concomitant and adjuvant temozolomide. Radiother Oncol. 2010;97(3):377-81.

17. Heye $\mathrm{T}$, Zausig N, Klauss M, et al. CT diagnosis of recurrence after pancreatic cancer: is there a pattern? World J Gastroenterol. 2011;17(9):1126-34.

18. Kapiteijn E, Marijnen CA, Nagtegaal ID, et al. Preoperative radiotherapy combined with total mesorectal excision for resectable rectal cancer. N Engl J Med. 2001;345(9):638-46.

19. Jones 3rd WE, Thomas Jr CR, Herman JM, et al. ACR appropriateness criteria(R) resectable rectal cancer. Radiat Oncol. 2012;7:161.

\section{Submit your next manuscript to BioMed Central and we will help you at every step:}

- We accept pre-submission inquiries

- Our selector tool helps you to find the most relevant journal

- We provide round the clock customer support

- Convenient online submission

- Thorough peer review

- Inclusion in PubMed and all major indexing services

- Maximum visibility for your research

Submit your manuscript at www.biomedcentral.com/submit

CBiomed Central 